\title{
Coupling of twin rectangular supersonic jets
}

\author{
By GANESH RAMAN ${ }^{1}$ AND RAY TAGHAVI ${ }^{2}$ \\ ${ }^{1}$ NYMA, Inc., Experimental Fluid Dynamics Section, \\ NASA Lewis Research Center Group, Brook Park, OH 44142, USA \\ ${ }^{2}$ Department of Aerospace Engineering, University of Kansas, \\ Lawrence, KS 66045, USA
}

(Received 21 March 1997 and in revised form 18 August 1997)

Twin jet plumes on aircraft can couple, producing dynamic pressures significant enough to cause structural fatigue. For closely spaced jets with a moderate aspect ratio (e.g. 5), previous work has established that two coupling modes (antisymmetric and symmetric) are kinematically permissible. However, the dynamics of twin-jet coupling have remained unexplored. In this paper a more fundamental assessment of the steady and unsteady aspects of twin-jet coupling is attempted. While we document and discuss the nozzle spacings and Mach numbers over which phase-locked coupling occurs, our concentration is much more on answering the following questions: (a) What mechanism causes the jets to couple in one mode or the other? (b) Why do the jets switch from one mode to another? (c) Are the two modes mutually exclusive or do they overlap at the transition point? Our results reveal, among many things, the following. (i) For very closely spaced twin jets in the side-by-side configuration phased feedback based on source to nozzle exit distance of adjacent jets does not fully explain the coupling modes. However, the 'null' phase regions surrounding the jets where the phase of an acoustic wavefront (arriving from downstream) does not vary appears to correlate well with the existence of the symmetric mode. When the 'null' regions of adjacent jets do not overlap antisymmetric coupling occurs and when they do overlap the jets couple symmetrically. We provide a simple correlation using a parameter $(\alpha)$ that can be used as a simple test to determine the mode of coupling. (ii) The switch from the antisymmetric to the symmetric mode of coupling appears to occur because of an abrupt shift in the effective screech source from the third to the fourth shock, which in turn causes the 'null' phase region surrounding the jets to grow abruptly and overlap. (iii) The two modes are mutually exclusive. Our results provide considerable insight into the twin-jet coupling problem and offer hope for designing twin-jet configurations that minimize damage to aircraft components.

\section{Introduction}

\subsection{Background and motivation}

Twin jet plumes on aircraft can couple, producing higher dynamic pressures in the inter-nozzle region, which in turn can cause sonic fatigue of external nozzle flaps. Hay \& Rose (1970) and Berndt (1984) have evidence that high dynamic pressures can cause nozzle or tail plane damage. Such damage is believed to be caused by the components of shock noise radiating upstream. Shock noise is made up of broadband (see Harper-Bourne \& Fisher 1973) and discrete tone (see Powell 1953) components that are produced by weak and strong interactions of the coherent structures with 
the shocks, respectively (Tam 1991). The discrete tone (screech) is produced by a feedback loop that involves a growing hydrodynamic disturbance that interacts with the shocks to produce a tone. The tone then propagates upstream to the jet exit and couples with the hydrodynamic disturbances. Although it was not established that screech was present in the flight tests of Berndt (1984), it was noted that the pattern of high dynamic pressures measured in the laboratory experiments matched the pattern of hardware damage that occurred during the flight test program. Thus, there is significant value in performing laboratory studies on twin supersonic jets that screech at a discrete frequency. The present fundamental research program on twin-jet coupling was undertaken to resolve numerous issues underlined by Tam \& Seiner (1987), and Morris (1990). Tam \& Seiner (1987) pointed to the need for documenting details of the antisymmetric mode of coupling and conditions under which this occurred. Morris (1990) emphasized the need for detailed experimental data on the twin-jet resonance problem.

Considerable work has been done on twin jet models to alleviate problems with the US Air Force B1-B and F15-E, at the NASA Langley Research Center (Seiner, Manning \& Ponton 1988; Seiner et al. 1992; Norum \& Shearin 1986); by the US Air Force (Shaw 1990; Walker 1990); and by McDonnell Douglas Aerospace (Wlezien 1987; Zilz \& Wlezien 1990). However, with the exception of the work by Zilz \& Wlezien (1990), very little has been done on twin rectangular jets. In addition, the above papers did not address details of the near acoustic field and the mechanism of coupling. Rectangular jets are currently of interest because of their use in military aircraft, and especially in situations requiring vectored thrust, stealth, or tail-less flight.

The present results are also intended to provide input to the screech simulation efforts of Cain et al. (1995), Cain \& Bower (1996), and Tam \& Shen (1998). It is hoped that the insight provided here will stimulate the development of twin-jet screech prediction methodology.

\subsection{Objectives}

Our experiments are directed towards helping answer some of the following specific questions about the interaction of twin jets: $(a)$ What mechanism causes the jets to couple in one mode or the other or not at all? (i.e. does the coupling occur through the acoustic field surrounding the jet or the hydrodynamic fields of the two jets?) (b) What causes the jets to switch from one mode to another? (c) How does the mode transition occur? (i.e. are the two dominant modes mutually exclusive, or do they overlap at the transition point?).

In addition to the above issues the paper documents the effect of varying the Mach number and nozzle spacing on the coupling and provides a detailed documentation of the near acoustic field. We also take into consideration that the problem is one of dynamic pressure loads in the inter-nozzle region. Most previous studies conducted time-averaged sound pressure level measurements. In the present work we used an ensemble-averaged time sequence over an entire screech cycle to assess the periodic loading. The rectangular nozzles in the present study were originally used in a stacked configuration for mixing enhancement and noise reduction studies (Rice 1995; Raman \& Taghavi 1996). In the present work the nozzles were used in the twin-jet exhaust configuration (see figure 1) to study details of the coupling effect.

The paper begins with a description of the twin-jet apparatus and the measurement and data analysis techniques $(\$ 2)$. Conditions for twin-jet coupling are discussed in $\$ 3.1$ followed by a characterization of the dominant modes of coupling (\$3.2). A 


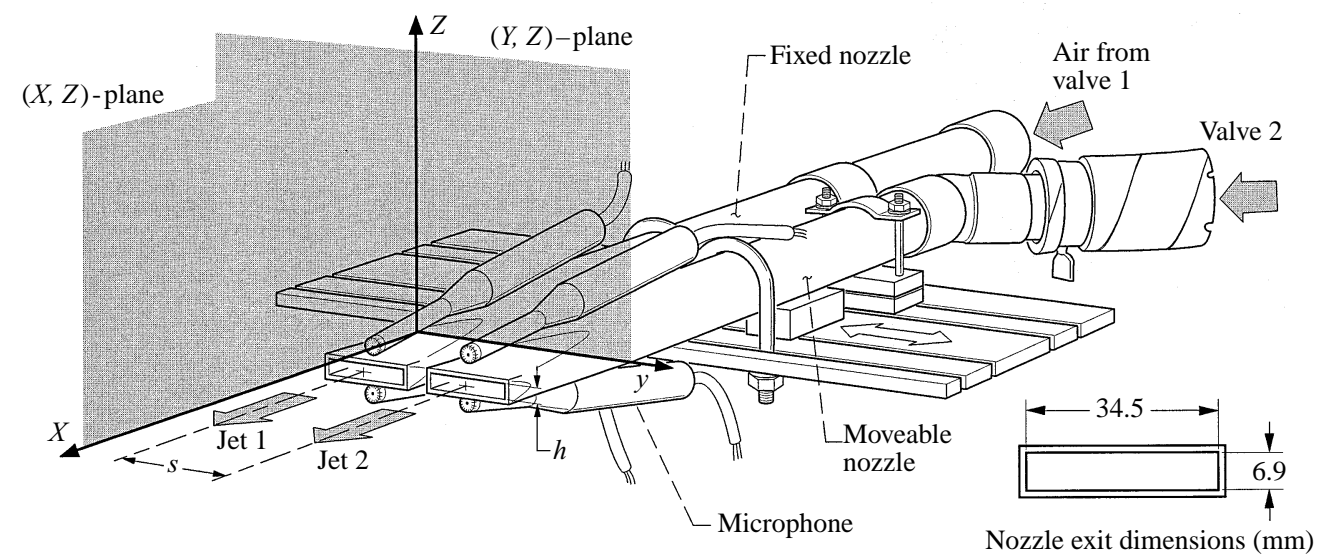

FIGURE 1. Sketch of twin supersonic rectangular nozzle set-up.

detailed depiction of the near acoustic field of coupled jets is provided in $\$ 3.3$. An explanation for why the twin jets select one coupling mode over the other and a parameter that can be used to determine the mode of coupling are given in $\$ 3.4$. Finally, observations on mode transition are included in $\S 3.5$.

\section{Experimental details}

Experiments were conducted in the continuous-flow supersonic jet facility at the NASA Lewis Research Center. The facility consisted of a plenum tank that included inflow conditioning and acoustic treatment. Two convergent nozzles were placed side by side with their narrow dimensions parallel and their long dimensions in the same plane (see figure 1). A positioning apparatus kept one of the nozzles fixed and the second nozzle was moved to achieve various inter-nozzle spacings. Microphones mounted on the nozzles monitored the acoustic phase relationship between the two jets. A detailed description of the facility, spark-schlieren system and microphone accuracy is given in Raman \& Taghavi (1996), and Raman (1997a) and will not be reiterated here.

A phase-locked time sequence over one screech cycle helps assess periodic changes in acoustic loading. Phase-averaged measurements (see Panda 1996) of near-field pressures were made using a reference microphone at the nozzle exit and a measurement microphone that traversed the entire $(Y, Z)$-plane (or $(x, z)$ - in some cases, see the sketch in figure 1). The signal from the reference microphone was band-pass filtered about the screech frequency to eliminate phase jitter. Data were ensemble-averaged over 100 oscillation cycles. The data acquisition rate $(200 \mathrm{kHz})$ was chosen so that phase-averaged distributions could be computed for 24-28 time steps per cycle (depending on the screech frequency). Phase averaged measurements on the $(Y, Z)$-plane included 350 data points, and the $\Delta Y$ and $\Delta Z$ values were both $0.635 \mathrm{~cm}$ - adequate to resolve the acoustic wavelength $(3.9$ and $3.5 \mathrm{~cm}$ for coupling mode I and II, respectively). For measurements on the $(X, Z)$ - and $(X, Y)$-planes, we used 322 and 391 data points, respectively, and $\Delta X, \Delta Y$, and $\Delta Z$ values of $0.635 \mathrm{~cm}$, which was also adequate to resolve the wavelength of the acoustic wave. 


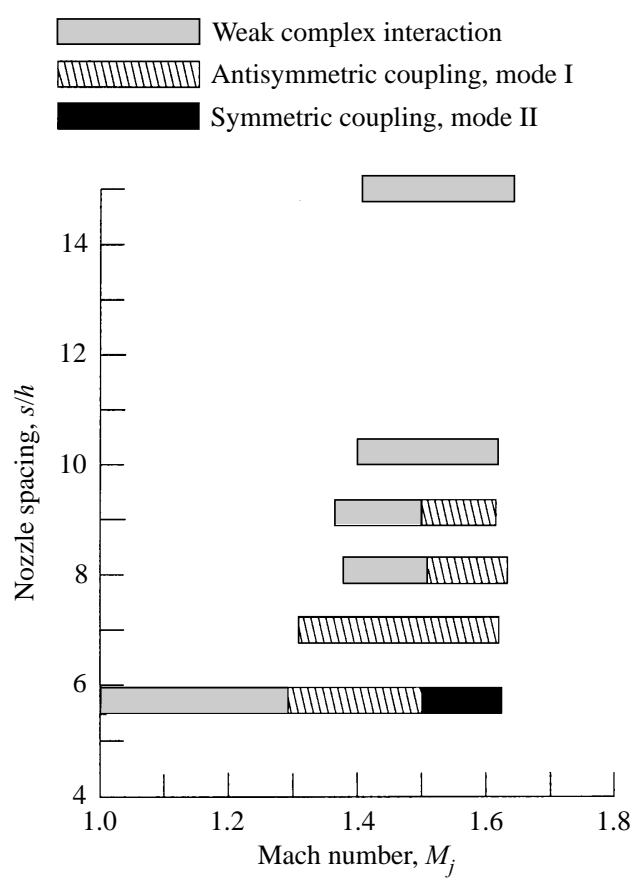

FIGURE 2. Coupling types for various twin-jet spacings and Mach numbers.

\section{Results and discussion}

\subsection{Conditions for twin-jet coupling}

The effect of varying nozzle spacing on the coupling of the twin rectangular jets was investigated. Figure 2 is a diagram showing the effect of varying the nozzle spacing (centre to centre) and the jet's fully expanded Mach number on the coupling. Coupling is defined as the phase locking of the screech instability of two adjacent jets. We observe only two out of the four possible modes determined by Zilz \& Wlezien (1990), Morris (1990), and Tam \& Thies (1993). They determined that when the jets flap (i.e. each jet oscillates antisymmetrically) in the normal direction $(X, Z$ plane), there are two preferred modes of coupling: one mode in which the jets are antisymmetric or out of phase $\left(180^{\circ}\right)$ with respect to each other and another where they are symmetric or in phase with respect to each other. In addition to the above modes two more modes are possible if the jets flap in the lateral direction $(X, Y$ plane). However, such lateral oscillations are observed only in low-aspect-ratio jets. Our jets have an aspect ratio of 5 and do not exhibit dominant lateral ( $X, Y$-plane) oscillations. Thus, the only possible oscillations for the two jets are in the normal $(X, Z)$ plane. In the present paper we refer to the two dominant coupling modes as mode I (antisymmetric) and mode II (symmetric).

From figure 2 it can be seen that a weak complex interaction (multiple tones present and phase-locked jet coupling absent) existed even when the jets were spaced $s / h=15$ apart. Note that $s$ represents the inter-nozzle spacing (centre to centre) and $h$ the smaller nozzle dimension. At both $s / h=10$ and 15 , weak complex interaction was observed. At $s / h=7.9$ and 8.9 , weak complex interaction was observed between $M_{j}=1.37$ and 1.5 , but phase-locked coupling (mode I) coupling existed (antisymmetric) between $M_{j}=1.50$ and 1.62 .

At $s / h=6.7$ there was mode I phase-locked coupling between $M_{j}=1.3$ and 1.62 . 
Finally, at $s / h=5.5$ there was weak complex interaction between $M_{j}=1.0$ and 1.3 , mode I phase-locked coupling between $M_{j}=1.3$ and 1.5, and mode II phase-locked coupling between $M_{j}=1.5$ and 1.62 . We will consider this last case in detail. Thus, at the closest spacing studied $(s / h=5.5)$ two strong modes of coupling existed: (i) antisymmetric (mode I), and (ii) symmetric (mode II).

At $s / h=5.5$ the jets stay coupled in the antisymmetric (mode I) from $M_{j}=1.3$ to 1.5 - even though the frequency changed from 10400 to 7488 and produced a change in the acoustic wavelength from $\lambda / h=4.78$ to 6.65 . If the two jets were operated at fully expanded Mach numbers such that the screech frequency produced by one of the jets is within about $3 \%$ of that of the other then phase-locked coupling occurred at small inter-nozzle spacings. In contrast a weak complex interaction occurred if one jet had a screech frequency that was $10 \%$ higher than that of the other (weak complex interaction was also achieved by operating the jets at different Mach numbers). Figure 3 shows examples of phase-locked coupling and weak complex interaction as we define them in this paper. Figures $3(a)$ and $3(b)$ are spectra from antisymmetric and symmetric phase-locked coupling cases (the microphones for jets 1 and 2 were located at $y / h=-2.75$ and +2.75 , respectively $(x / h=0, z / h=1$ for both cases)). To obtain the weak complex interaction cases we operated the two jets at different Mach numbers $M_{j}=1.525$ and 1.504 for the data in figure $3(c)$ and 1.59 and 1.57 for the data in figure $3(d))$. In figure $3(c)$ note that jet 1 had a dominant screech frequency of $7234 \mathrm{~Hz}(152 \mathrm{~dB})$, whereas jet 2 had a dominant screech frequency of $7983 \mathrm{~Hz}(156$ $\mathrm{dB})$. Note also that besides these two frequencies there are some sidebands and other tones that could be produced by sum and difference frequencies. The discussion of these is not within the scope of the present work (see Walker, Gordeyev \& Thomas 1995 that deals with a screeching jet with multiple tones). Further evidence of weak complex interaction is presented in figure $3(d)$. The reasoning is the same as that offered with figure $3(c)$.

Figure 4 shows spectra and coherence between microphones on adjacent nozzles for various inter-nozzle spacings. Spectra shown in figure $4(a-d)$ are from a microphone placed on nozzle 1 (see figure 1). Spectra from the microphone on nozzle 2 are virtually identical and thus omitted. The coherence between the two microphone signals is shown in figure $4(e-h)$. At very large nozzle spacings $(s / h=15)$ the measurements (figure $4 e$ ) indicate that the signals from jets 1 and 2 have low coherence. In addition, at such large nozzle spacings the two jets produce screech tones at slightly different frequencies, and this is seen as two closely spaced peaks in the coherence. As the internozzle spacing is decreased only a single peak is visible in the coherence measurement and the value of the coherence function approaches 1 (figure $4 f-h$ ). Another obvious change that is observed as the inter-nozzle spacing is decreased is the relatively high coherence at frequencies lower than the screech frequency. It is clear that there are coherent pressure fluctuations in a band of frequencies that are in the range of the hydrodynamic instability modes growing in the jet.

\subsection{Dominant modes of coupling}

The two modes will now be described using the flow visualization photographs of figure 5 and the data of figure 6 . Schlieren flow visualization of the two modes of coupling is shown in figure $5(a-f)$. The three views shown include the edge view (figure $5 a, d$ ), a $30^{\circ}$ rotation from the edge view (figure $5 b, e$ ) and a $90^{\circ}$ rotation from the edge view that provides the plan view (figure $5 c, f$ ). For both modes of coupling the jets flap in the plane $(X, Z)$ seen in figure $5(a, d)$. For mode I coupling the two jets flapped $180^{\circ}$ out of phase with respect to each other. Since one jet hides the 

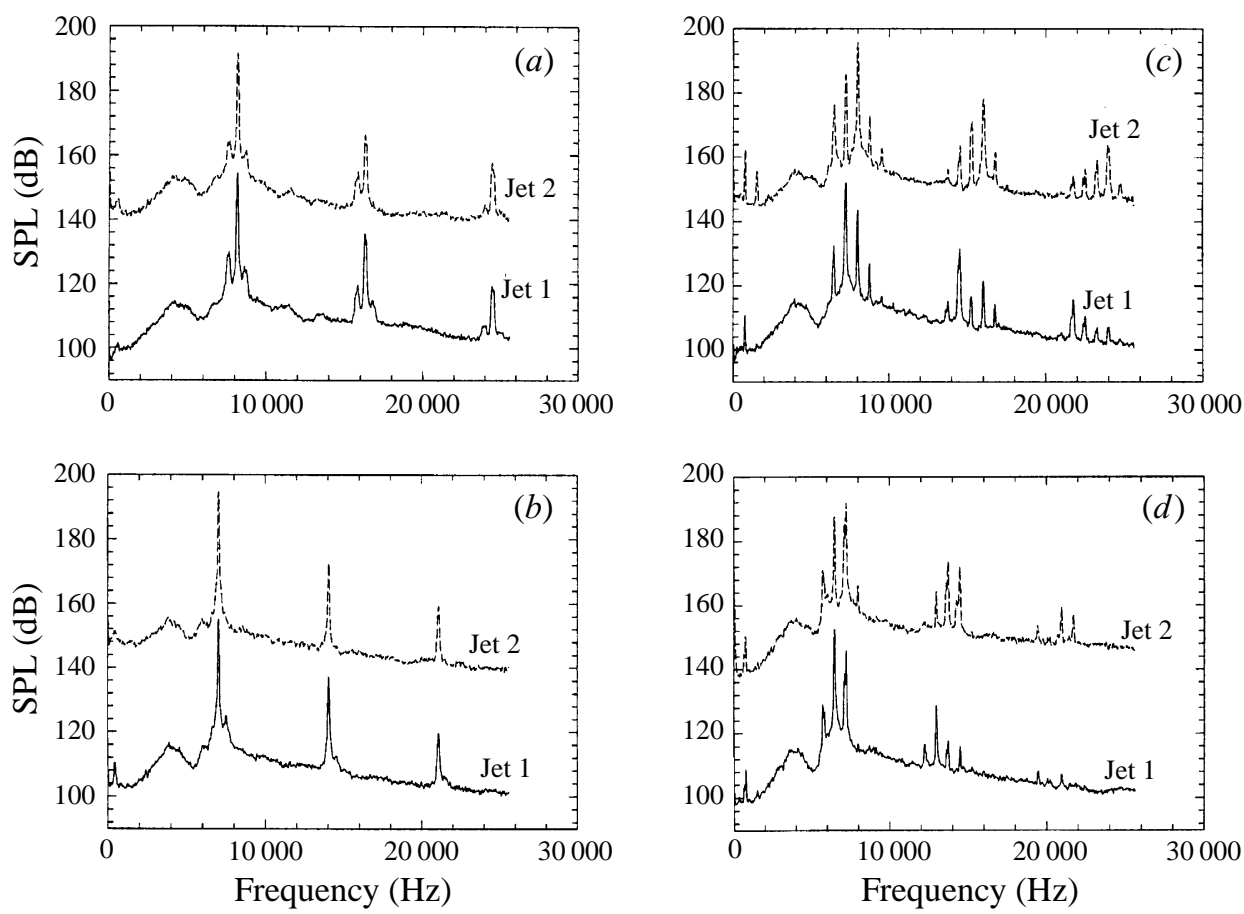

FIGURE 3. Spectra depicting phase-locked coupling and weak complex interaction of twin jets. $s / h=5.5$, dashed curves are vertically offset by $40 \mathrm{~dB}$. (a) Phase-locked coupling mode I, $M_{j}=1.48$, (b) phase-locked coupling mode II, $M_{j}=1.55,(c)$ weak complex interaction obtained by operating jets at $M_{j}$ of 1.525 and $1.504,(d)$ weak complex interaction obtained by operating jets at $M_{j}$ of 1.59 and 1.57 .

other in the edge view (figure 5a), the picture provides an integrated image of the two out of phase sinuous oscillations. In the case of mode II, the jets were coupled in phase and the edge view shows a single unified oscillation (figure $5 d$ ). In the $30^{\circ}$ view sinuous oscillations are seen between the fourth and fifth shock cells. These oscillations are out of phase in figure 5(b) (mode I) and in phase in figure 5(e) (mode II). The plan view (figure $5(c, f)$ ) does not reveal details of the oscillations but is included to document the spanwise shock-cell structure during coupling.

Figure 6 compares the frequency and amplitude of single and coupled jets over the entire Mach number range. The figure also depicts the relative phase variation between the two coupled jets. Figure 6(a) shows that when two jets are operated individually the screech frequency versus Mach number curve could be slightly different if there are manufacturing imperfections. When both jets were operated simultaneously the twin jets picked a screech frequency that was different from the frequency of either jet operated individually. However, the frequency of the coupled jets was closer to the frequency of jet 1 than that of jet 2 for mode I coupling (perhaps because jet 1 had a higher screech amplitude than jet 2, see figure $6 b$ ). In other words the coupling produces a new feedback loop at a new frequency that overrides the original feedback loops of the two jets. Note also that a small shift in frequency occurred (figure $6 a$ ) when the coupled twin jets switched to mode II (at a fully expanded Mach number of 1.5 ).

The screech amplitude data shown in figure $6(b)$ were obtained using microphone 2 located midway between the two nozzles $(x / h=0, y / h=0, z / h=1)$ for the 

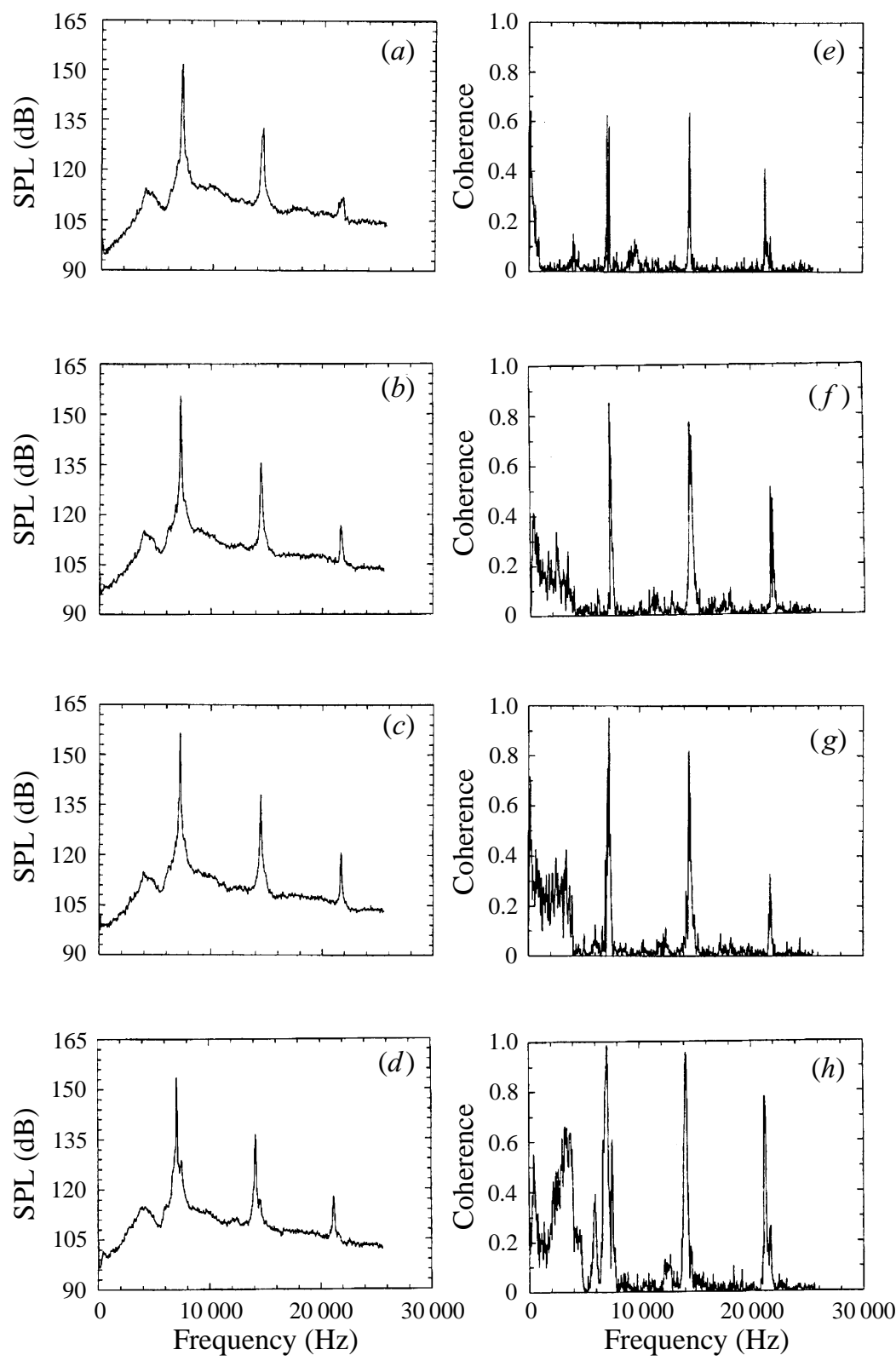

FigURE 4. Spectra and coherence between microphone signals from adjacent nozzles for various inter-nozzle spacings. $M_{j}=1.55$. $(a, e) s / h=15,(b, f) 9,(c, g) 8,(d, h) 5.5$.

twin coupled jets and for the case where only one jet was operated. It is clear that the sound pressure levels for mode I coupling were about $15 \mathrm{~dB}$ lower than the single jet cases. Note that these levels are lower than what could be obtained by a mere difference of the sound pressure levels of the two individual jets. Thus screech cancellation produced by mode I coupling in the inter-nozzle region is very strong. Although a switch from mode I to II increased the sound pressure levels by $20 \mathrm{~dB}$, mode II did not exhibit significant screech amplification (i.e. the levels were not much higher than the sum of the sound pressure levels of the individual jets). 


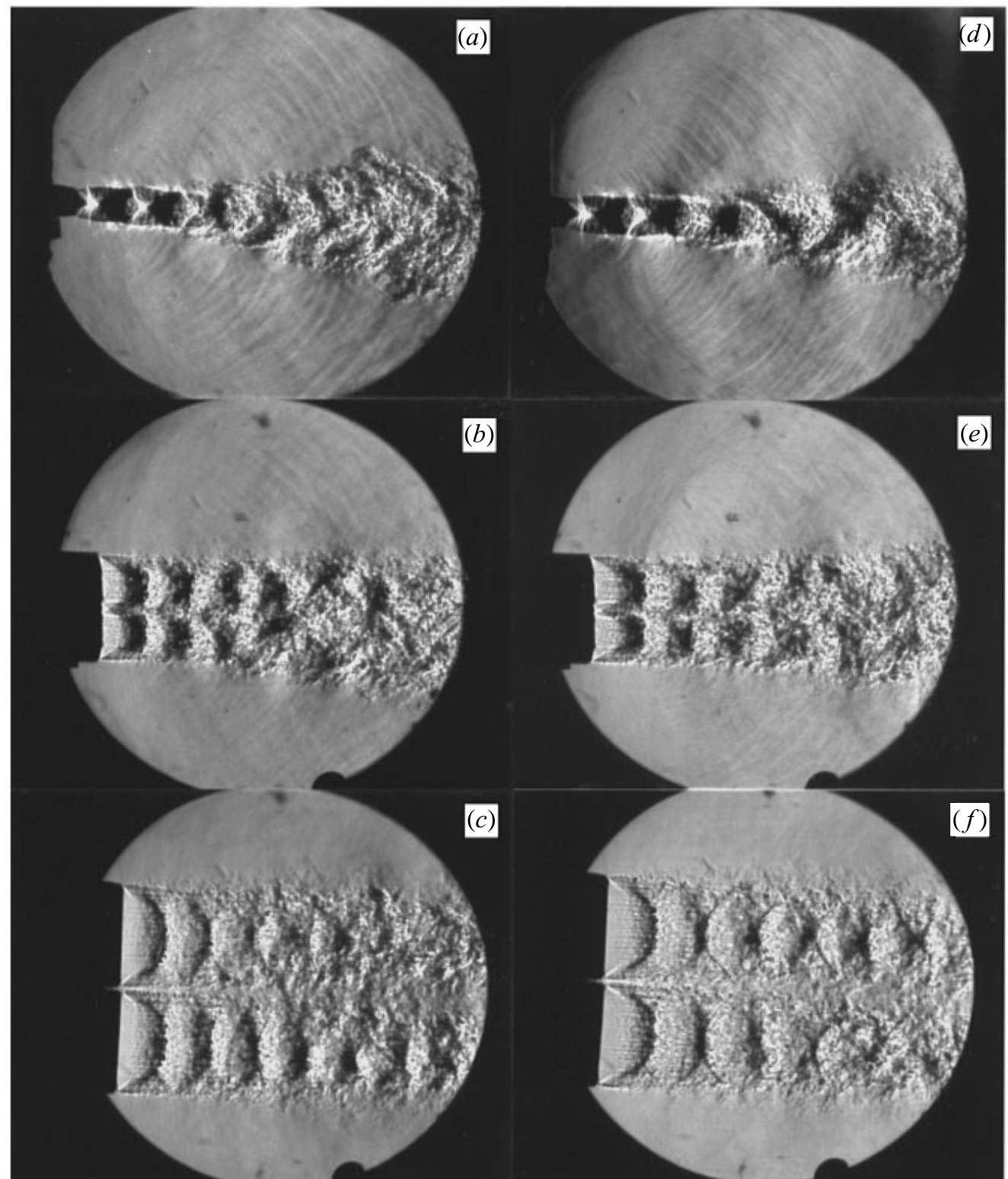

FIGURE 5. Schlieren flow visualization of the two modes of coupling. $(a-c)$ mode I, $(d-f)$ mode II. $(a, d)$ edge view (smaller dimension) of the jets, $(b, e)$ view after rotating nozzles by $30^{\circ}$ from edge view, $(c, f)$ plan view (larger dimension) of the jets.

The phase difference shown in figure 6(c) was between microphones 1 and 3, and this measurement indicated whether the jets were coupled antisymmetrically or symmetrically. A phase difference of $180^{\circ}$ between symmetrically located microphones on nozzles 1 and 3 indicated that they were coupled out of phase up to an $M_{j}$ of 1.5 , beyond which a mode switch occurred and the jet coupling became symmetric. Details of the difference between the two modes of coupling will be described in later subsections.

A brief discussion of the differences between circular and rectangular screeching 

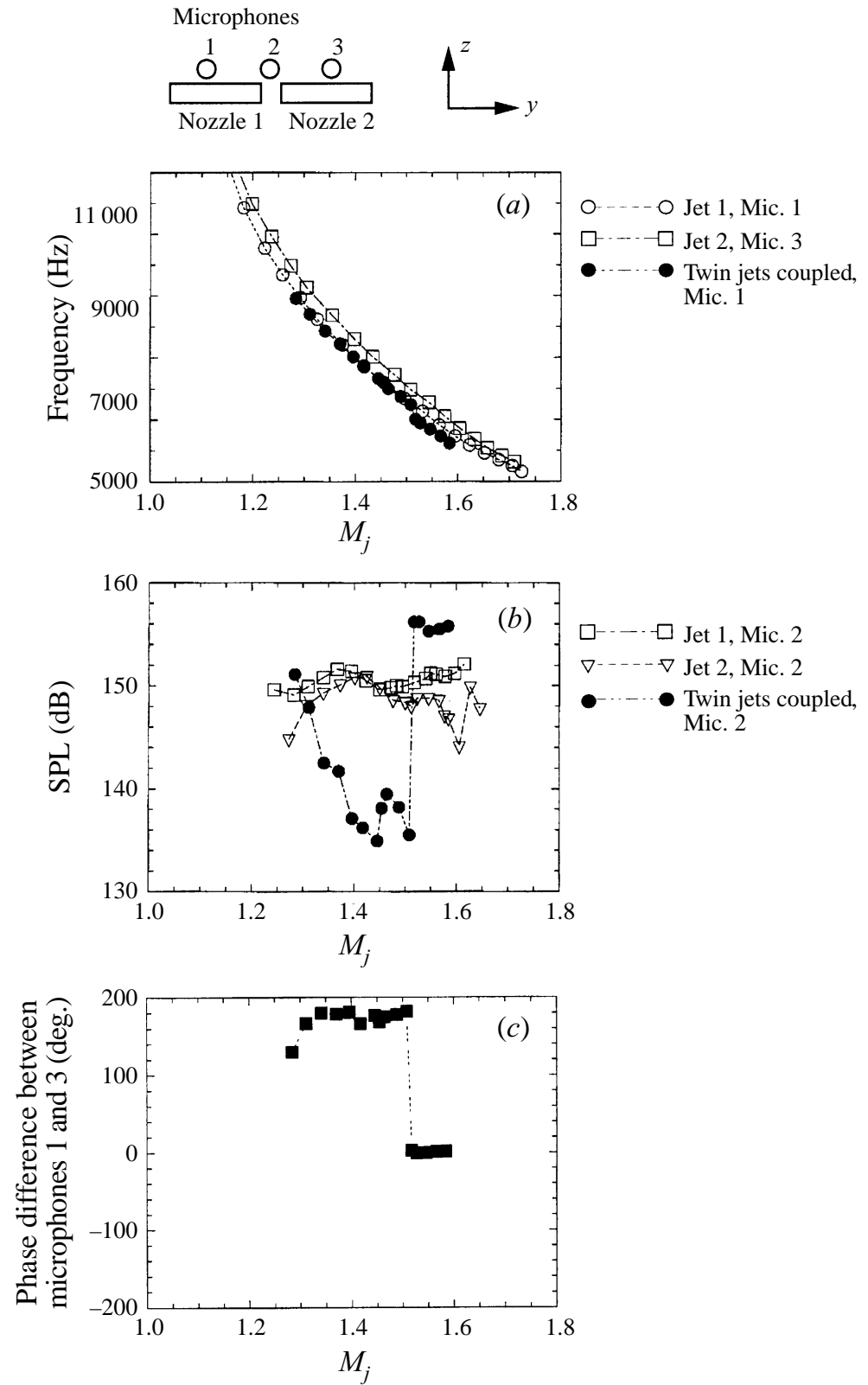

FiguRE 6. Characteristics of single and twin coupled jets versus Mach number. $s / h=5.5$. (a) Screech frequency versus Mach number, $(b)$ screech tone amplitude versus Mach number, (c) phase difference between jets 1 and 2 versus Mach number.

jets is in order before concluding this subsection. A single circular jet exhibits several modes of screech (Powell 1953) often referred to as stages A-E. In contrast, the single rectangular jet (aspect ratio $\geqslant 5$ ) has one dominant mode. When twin-circular jets are operated simultaneously, the jet modes can couple in more than one way, depending on nozzle spacing and the Mach number. However, the sound pressure level in the inter-nozzle region rises dramatically when the B (helical) modes of the two jets couple (Norum \& Shearin 1986). In comparison, the present twin-rectangular-jet experiments 
exhibit coupling in two modes (I and II as described earlier). Mode I reduces the sound pressure in the inter-nozzle region and mode II augments it.

\subsection{The near acoustic field of coupled jets}

Focus now shifts to the near acoustic field produced by the two types of coupling. Detailed measurements were made on the two planes $(Y, Z$ and $X, Z)$ described in figure 1 . The $(Y, Z)$-plane was located at $x / h=0$, and the $(X, Z)$-plane was located at $y / h=0$. Figure $7(a-d)$ shows the magnitude and relative phase on the $(Y, Z)$-plane. The magnitude (expressed as SPL in $\mathrm{dB}$ ) was obtained by moving a microphone over the entire $(Y, Z)$-plane. The relative phase was obtained from the cross-spectral phase between the traversing microphone and a reference microphone. For mode I coupling (figure $7 a$ ), the two jets displayed an amplitude minimum between the two nozzles. The relative phase plot (figure $7 b$ ) showed a $180^{\circ}$ phase difference between symmetric locations on either side of $y / h=0$. In contrast, for mode II coupling (figure 7c) the acoustic signature on the $(Y, Z)$-plane indicated that the two jets had combined into one with the maximum sound pressure level occurring in the inter-nozzle region. The phase plot (figure $7 d$ ) shows a zero degree phase difference between symmetric locations on either side of $y / h=0$.

Although the problem at hand is one of dynamic pressure loads, most previous studies have focused on average sound pressure levels. Phase-averaged measurements on the $(Y, Z)$-plane for mode I and II coupling are shown in figures 8 and 9 , respectively. The solid and dashed lines represent positive and negative phases of the screech cycle, respectively. Note that the pressure waves were propagating upstream (normal to the $(Y, Z)$-plane shown in figures 8 and 9 ) and what one sees in figures 8 and 9 is a projection on the $(Y, Z)$-plane at various phases of the screech cycle. From figures 8 and 9 it is clear that the pressure waves were antisymmetric and symmetric, respectively (as evidenced by the dual regions of alternating positive and negative pressures, and by single regions of alternating positive and negative pressures, respectively).

We now shift our attention to the $(X, Z)$-symmetry plane. Detailed features of pressure waves during feedback can be seen from the phase-averaged data of figures 10 and 11 for coupled jets in modes I and II. Similar data on the instantaneous pressure field of a round jet were given in Westley \& Woolley $(1969,1970)$, and studied in more detail recently by Panda (1996). For mode I coupling (figure 10) where the pressure is minimized on the $(X, Z)$-plane, there are weak axially $(x)$ propagating and vertically $(z)$ propagating waves (seen as islands of alternating solid and dashed lines). However, for mode II coupling, where the pressure is maximized on the $(X, Z)$-plane, the axially propagating waves dominate (figure 11). From the contour values of figures 10 and 11 (not shown) and the corresponding single jet cases (also not shown in the interest of conciseness) the maximum and minimum pressures on the symmetry plane $(X, Z)$ for the various cases are shown in figure 12. These maxima and minima could have occurred at different locations on the plane for the various cases. However, this aspect is not considered here. It is clear that mode I coupling decreased the spread between the maximum and minimum (figure 12a) and mode II coupling increased it (figure 12b). More interestingly, note that the single jet cases at $M_{j}$ values that produce mode I coupling (figure 12a) and mode II coupling (figure 12b) were quite different.

Figure 13 compares the phase-averaged near acoustic field (at one phase of the reference signal) in the $(X, Y)$-plane (see figure 1 for coordinates), for single and coupled twin jets. The $(X, Y)$-plane was located at $z / h=3$. The data shown in 

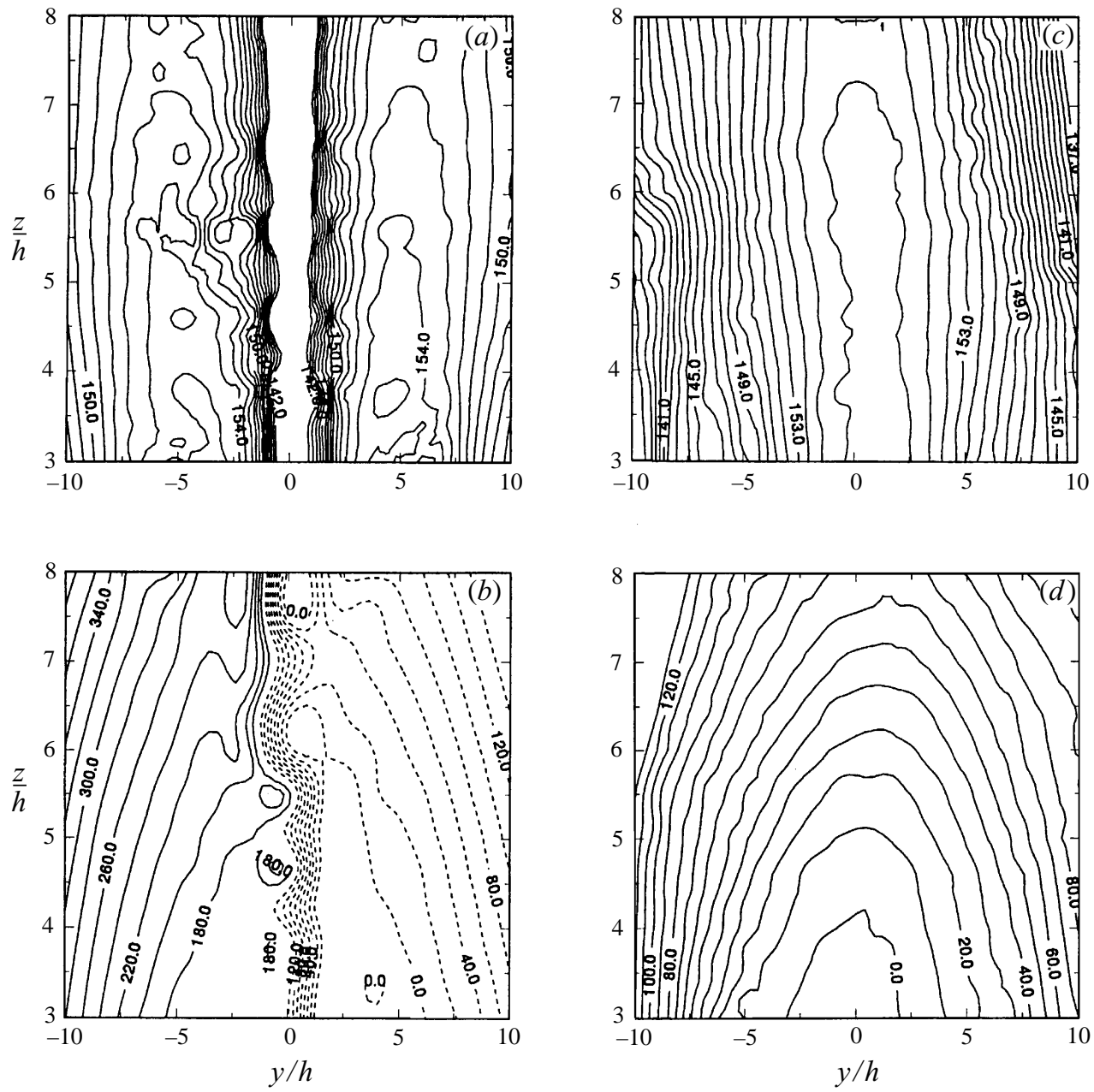

FiguRE 7. Time-averaged near acoustic field in the $(Y, Z)$-plane for twin coupled jets. $s / h=5.5$. $(a, c)$ Amplitude, $(b, d)$ relative phase, $(a, b)$ mode I coupling, $M_{j}=1.48,(c, d)$ mode II coupling, $M_{j}=1.55$.

figure $13 a, b$ were obtained by operating a single jet at fully expanded Mach numbers that produced the two modes of coupling when both jets were operated simultaneously. The instantaneous pressure patterns for the two single jet cases (figure 13a,b) do not show notable differences between them. For the coupled jet cases, mode I coupling (figure 13c) clearly displays that the sources of adjacent jets are anti-phase in $y$ over the entire axial extent of the jets. In contrast, for mode II coupling (figure $13 d$ ) the sources of both jets were in-phase and the combined picture resembled that of a single jet. Finally, we hope that the detailed documentation of the near acoustic field provided in this subsection will serve as benchmark data for comparison with numerical simulations.

\subsection{Coupling mechanism}

Tam \& Thies (1993) studied the wave-modes in a rectangular jet that were given by the eigensolutions of the linearized governing equations. They found that there were four linearly independent families of eigensolutions that were invariant to 

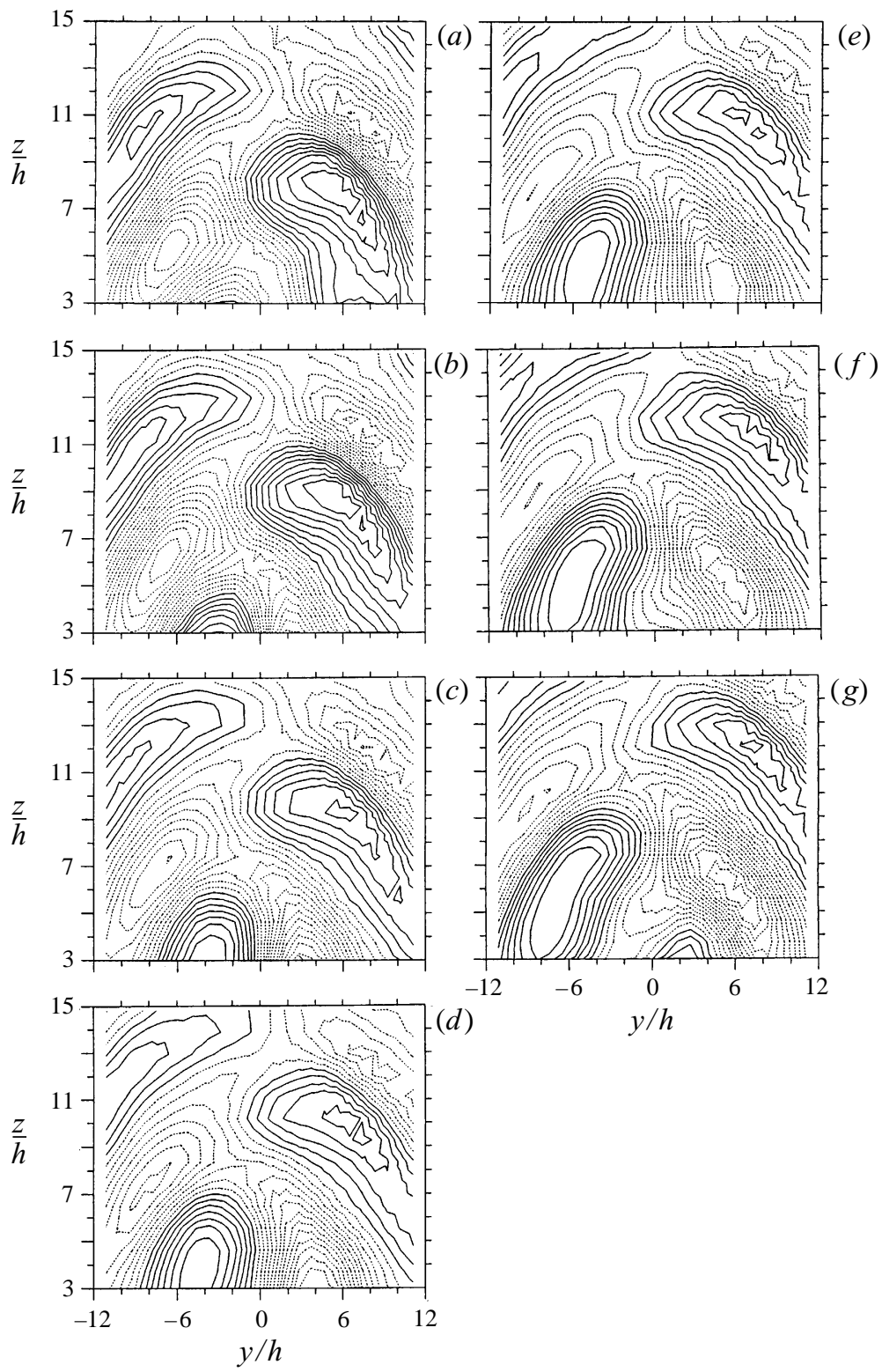

$(d)$

$y / h$

FiguRe 8. Phase-averaged near acoustic field in the $(Y, Z)$-plane for twin jets coupling in mode I. $M_{j}=1.48, s / h=5.5$. Phase difference from frame to frame is $30^{\circ}$. Parts $(a-g)$ represent half a cycle. Solid and dashed lines represent positive and negative phases of the screech cycle, respectively.

certain transformations. The four families that they identified as being kinematically permissible were the same as the four possible coupling modes identified by Morris (1990) and Zilz \& Wlezien (1990) (i.e. modes that were antisymmetric or symmetric in the normal $(z)$ and lateral $(y)$ directions).

In our experiment each rectangular screeching jet (aspect ratio $=5$ ) exhibited what Tam \& Thies (1993) termed a 'family 2 ' instability (i.e. flapping in the $(X, Z)$ plane). The coupled twin jets in our experiments (also having an aspect ratio of 5) exhibited either a 'family 4' (mode I of this paper, where each jet was antisymmetric 

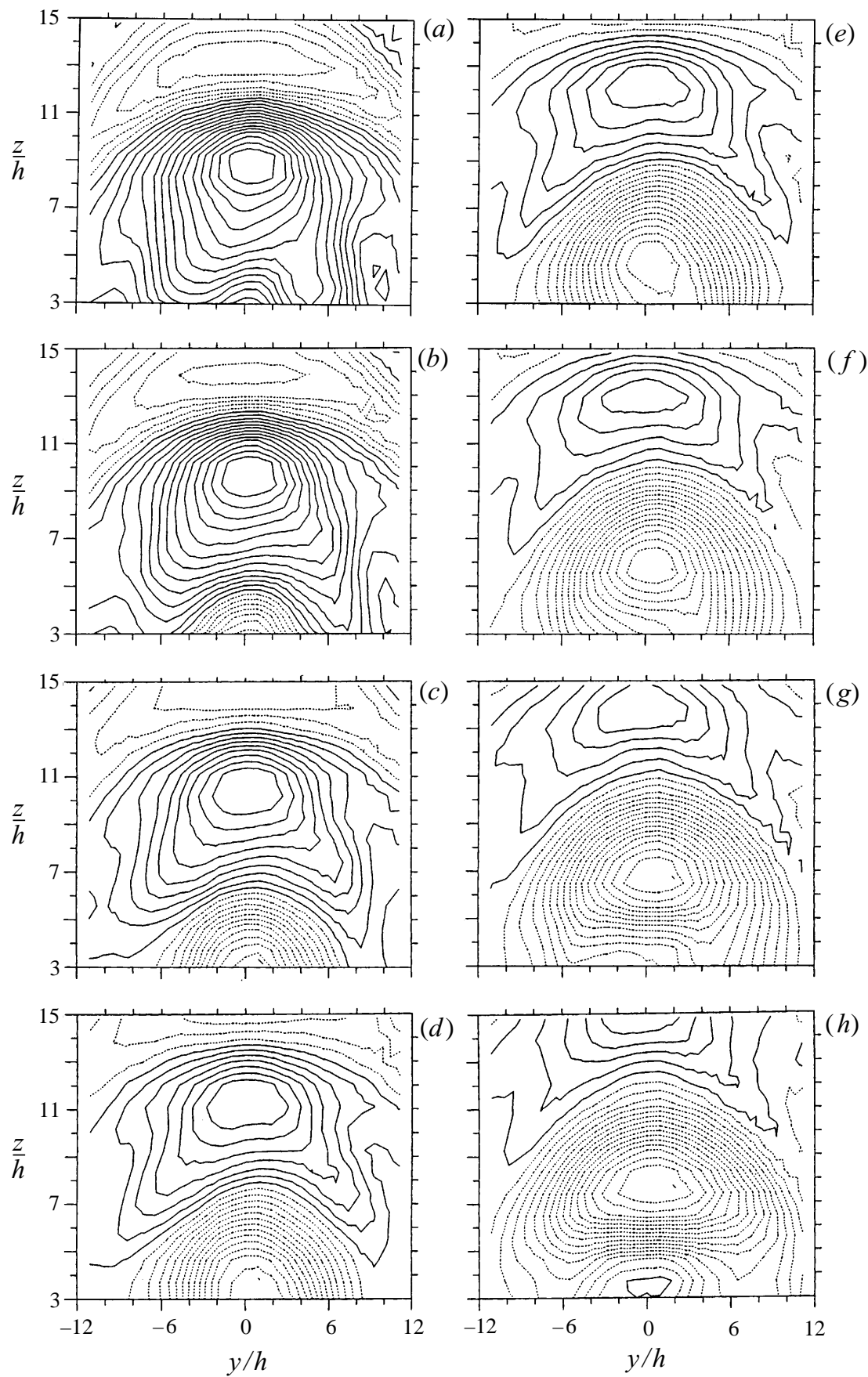

FiguRE 9. Phase-averaged near acoustic field in the $(Y, Z)$-plane for twin jets coupling in mode II. $M_{j}=1.55, s / h=5.5$. Phase difference from frame to frame is $25^{\circ}$. Parts $(a)-(h)$ represent half a cycle. Solid and dashed lines represent positive and negative phases of the screech cycle, respectively. 

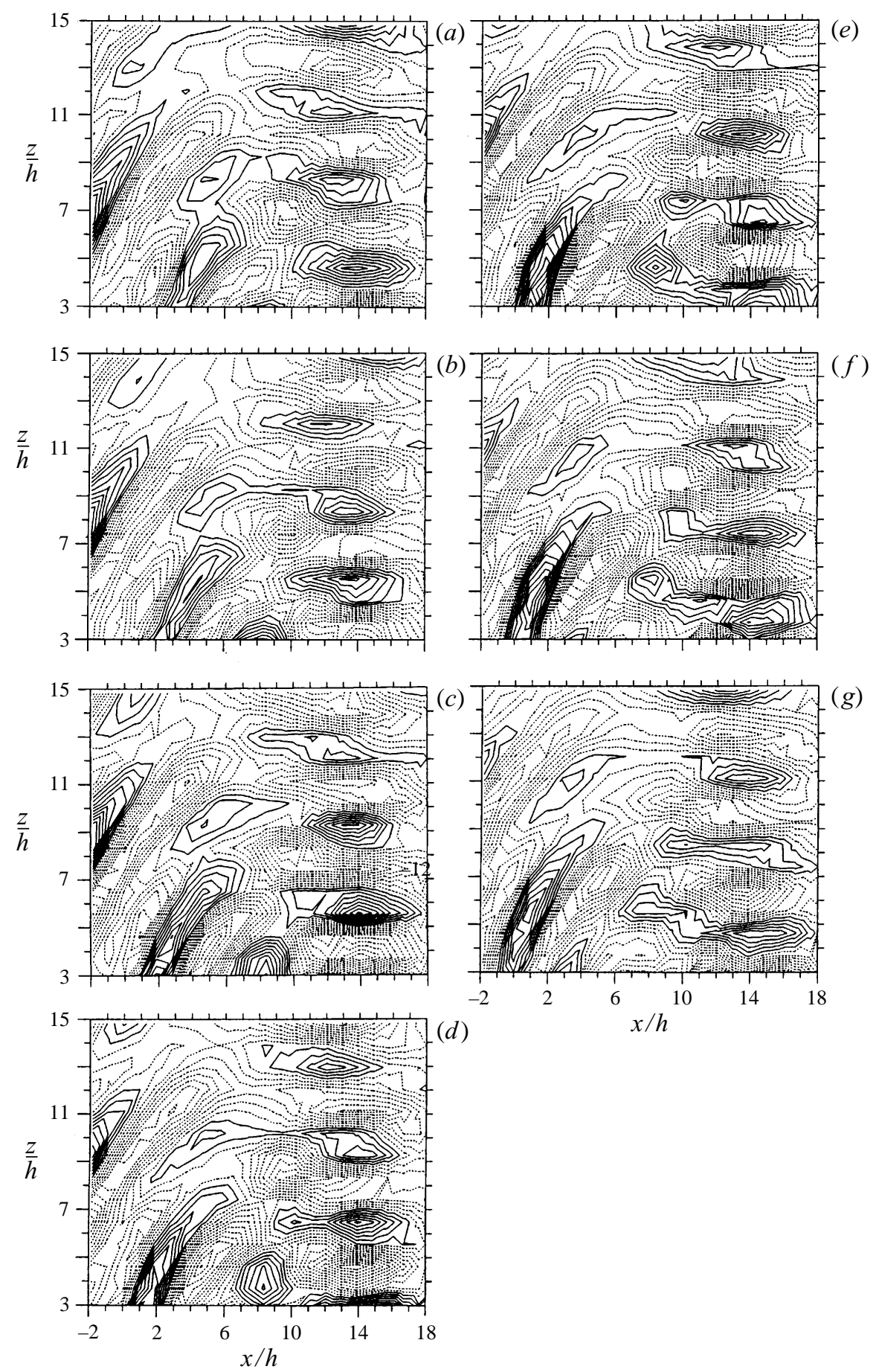

Figure 10. Phase-averaged near acoustic field in the $(X, Z)$-plane for twin jets coupling in mode I. $M_{j}=1.48, s / h=5.5$. Phase difference from frame to frame is $30^{\circ}$. Parts $(a-g)$ represent half a cycle. Solid and dashed lines represent positive and negative phases of the screech cycle, respectively. 

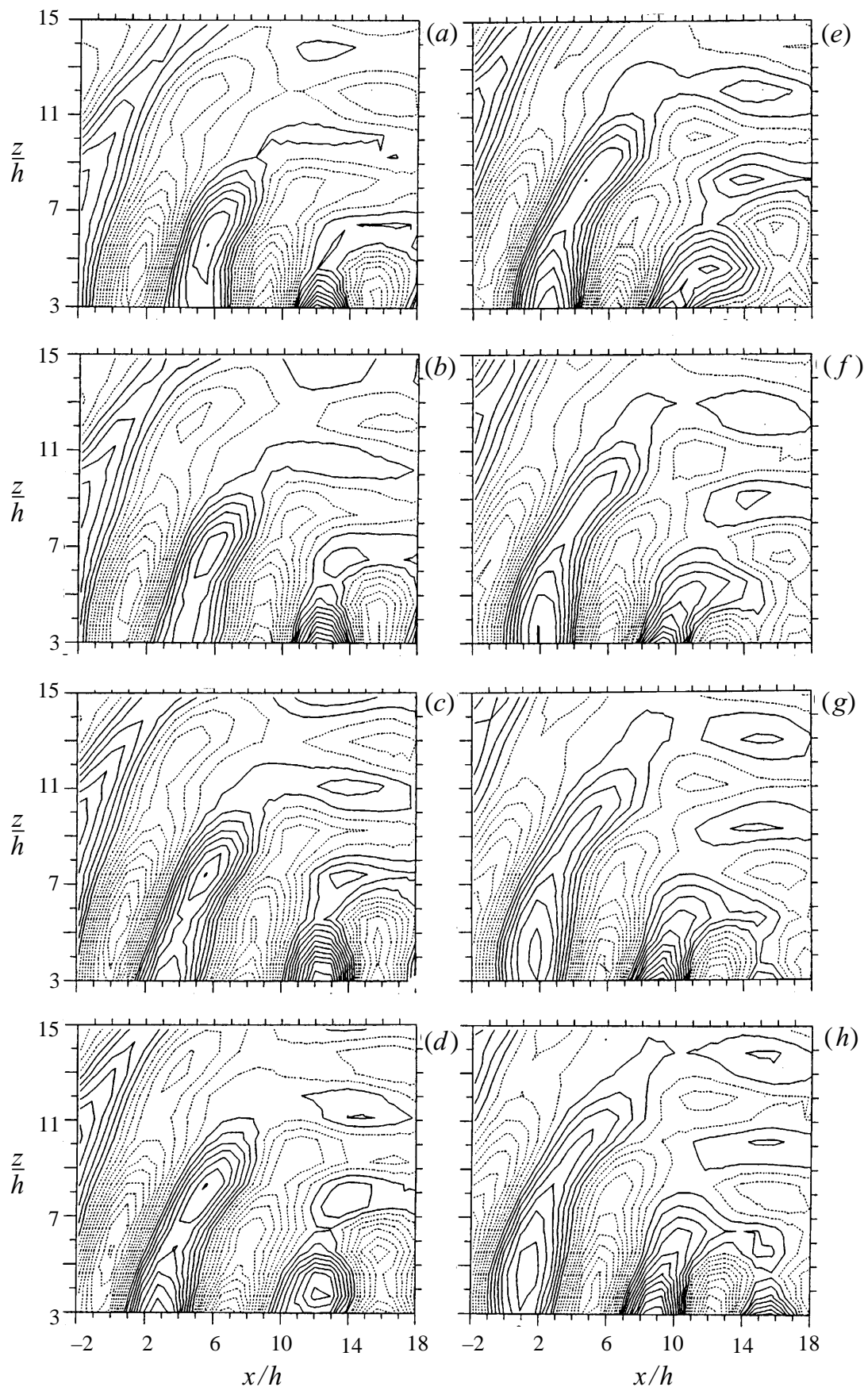

FiguRE 11. Phase-averaged near acoustic field in the $(X, Z)$-plane for twin-jets coupling in mode II. $M_{j}=1.55, s / h=5.5$. Phase difference from frame to frame is $25^{\circ}$. Parts $(a-h)$ represent half a cycle. Solid and dashed lines represent positive and negative phases of the screech cycle, respectively. 

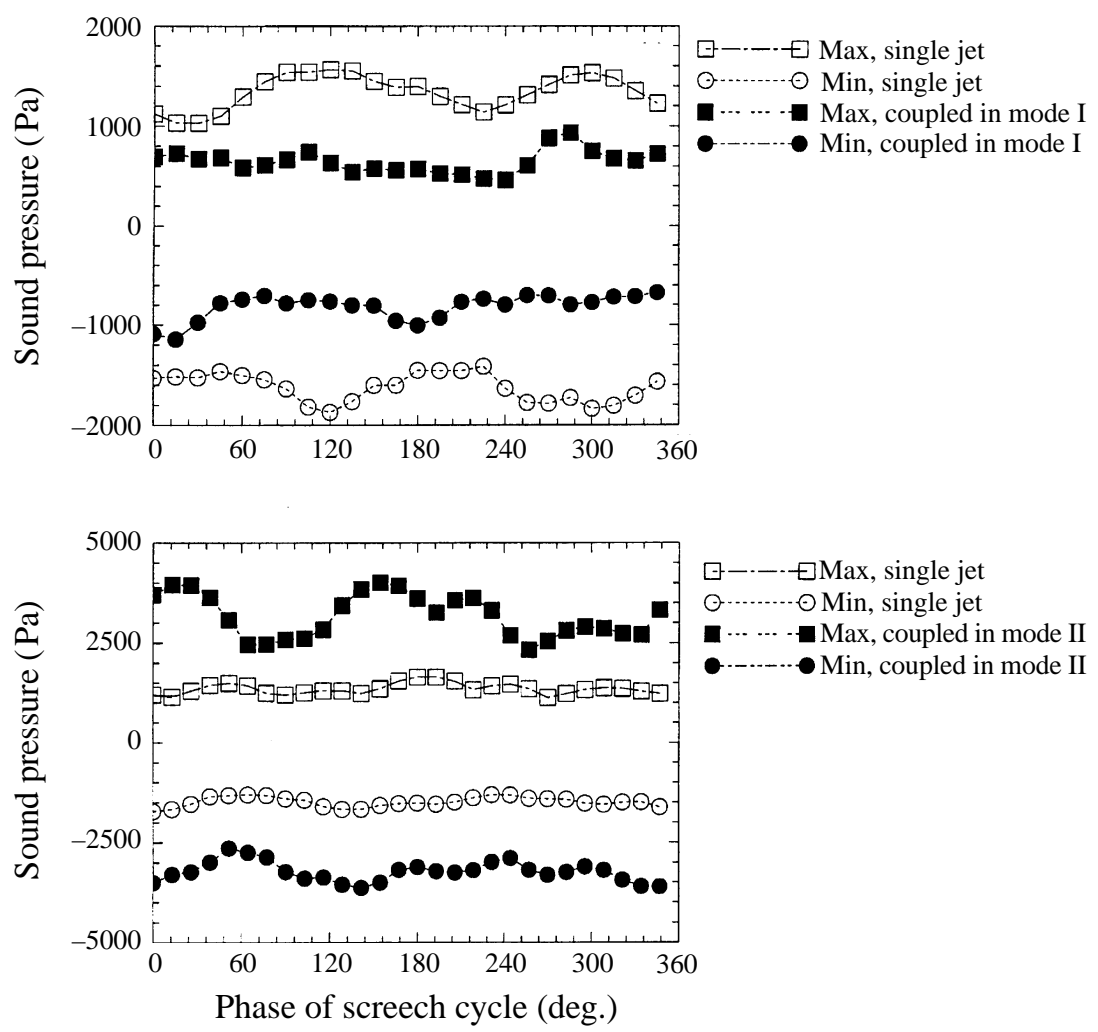

FIGURE 12. Maximum and minimum phase-averaged pressure loads on the $(X, Z)$-plane for single and twin coupled jets during one screech cycle. (a) $M_{j}=1.48$. Mode I coupling, $(b) M_{j}=1.55$, Mode II coupling.

with respect to the other) or a 'family 2' (mode II of this paper, where each jet was symmetric with respect to the other) instability. Thus, the two modes that were known to be kinematically permissible for the aspect ratio used were observed. The issue now is what mechanism caused the twin jets to choose one coupling mode over the other?

To answer the above question we recall that when two jets are in close proximity, each becomes a sound source for the feedback loop of the other jet. The phase relationship between the source of each jet and the nozzle exit of the other then determines the mode of coupling (Wlezien 1987). This idea was confirmed in earlier work (Rice 1995; Raman \& Taghavi 1996) on multiple rectangular jets in a stacked configuration. The present work differs from the Raman \& Taghavi (1996) work in two important ways. In the present work the jets are in a side-by-side configuration (see figure 1) and the inter-nozzle spacing is very small. For the present configuration phased feedback from adjacent jets does not explain the coupling modes or the sudden switch from mode I to mode II. This is clear from the fact that the jets remained coupled in mode I from $M_{j}=1.3$ to 1.5 even though the screech frequency changed from 10400 to 7488 and produced a corresponding change in the acoustic wavelength from $\lambda / h=4.78$ to 6.65 . Further, after the mode switch (from I to II) occurred at $M_{j}=1.5$, the jets remained coupled in mode II even as the Mach number was increased further.

The data of figures 14 and 15 will be used to examine how the jets choose the 

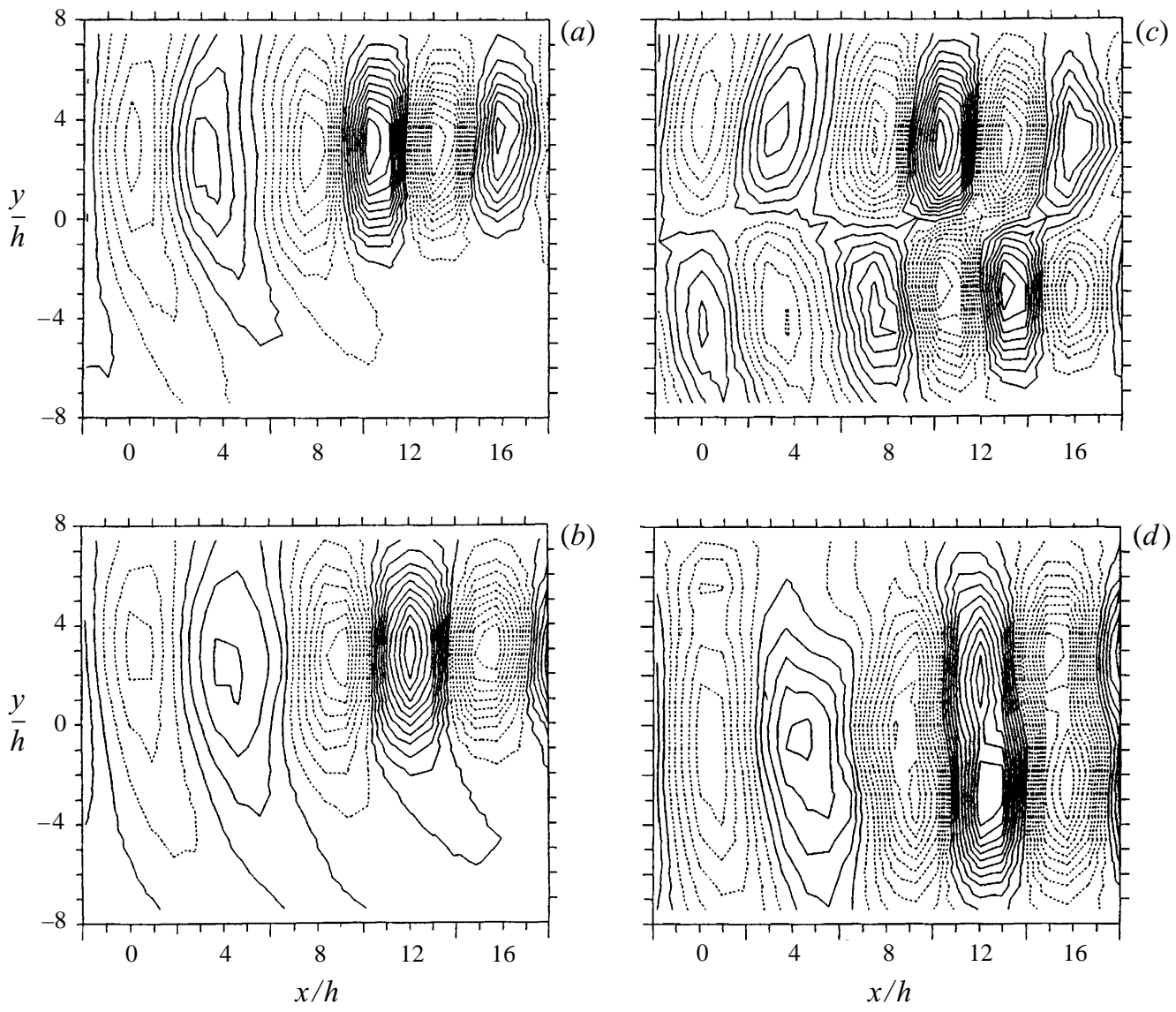

FIGURE 13. Comparison of the phase-averaged near acoustic field in the $(X, Y)$-plane for single and coupled twin jets. (a) Single jet, $M_{j}=1.48,(b)$ single jet, $M_{j}=1.55,(c)$ twin jets coupled in mode I, $M_{j}=1.48,(d)$ twin jets coupled in mode II, $M_{j}=1.55$.

coupling mode. Figure $14(a, b)$ shows data similar to that of figure $7(a, c)$ but for a single nozzle that was operated at fully expanded jet Mach numbers $\left(M_{j}\right)$ that produced the two types of coupling. Although only one nozzle (located at $y / h=2.75$ ) was operated the time-averaged amplitude was measured over the entire twin-jet coupling region on the $(Y, Z)$-plane. Figure 14(a) shows that at the fully expanded jet Mach number $\left(M_{j}\right)$ that produced mode I coupling the peak amplitude was closer to the outer edge of the nozzle lip $(z / h=3)$. In contrast, it can be seen from figure $14(b)$ that at the $M_{j}$ that produced mode II coupling, the peak amplitude (island) was located farther away from the outer edge of the nozzle lip $(z / h=7)$.

Figure 15 shows the relative phase corresponding to the amplitude data of figure $14(a, b)$ along the $y / h=2.75$ line in the near nozzle region. Particularly important here is the 'null' phase region surrounding the jets ( $\mathrm{n} 1$ and $\mathrm{n} 2$ in figure 15) where the phase of an acoustic wavefront (arriving from downstream) does not vary over a small distance. Note that the 'null' region is defined as the extent in $z$ over which the phase change is $10^{\circ}$ (our measurement accuracy is $\pm 5^{\circ}$ ). The existence of this 'null' region and its growth with $M_{j}$ appears to occur (at least to some extent) because of the movement of the equivalent source of screech further downstream that causes the wavefronts arriving at the nozzle exit plane to be flatter in the near nozzle region. 

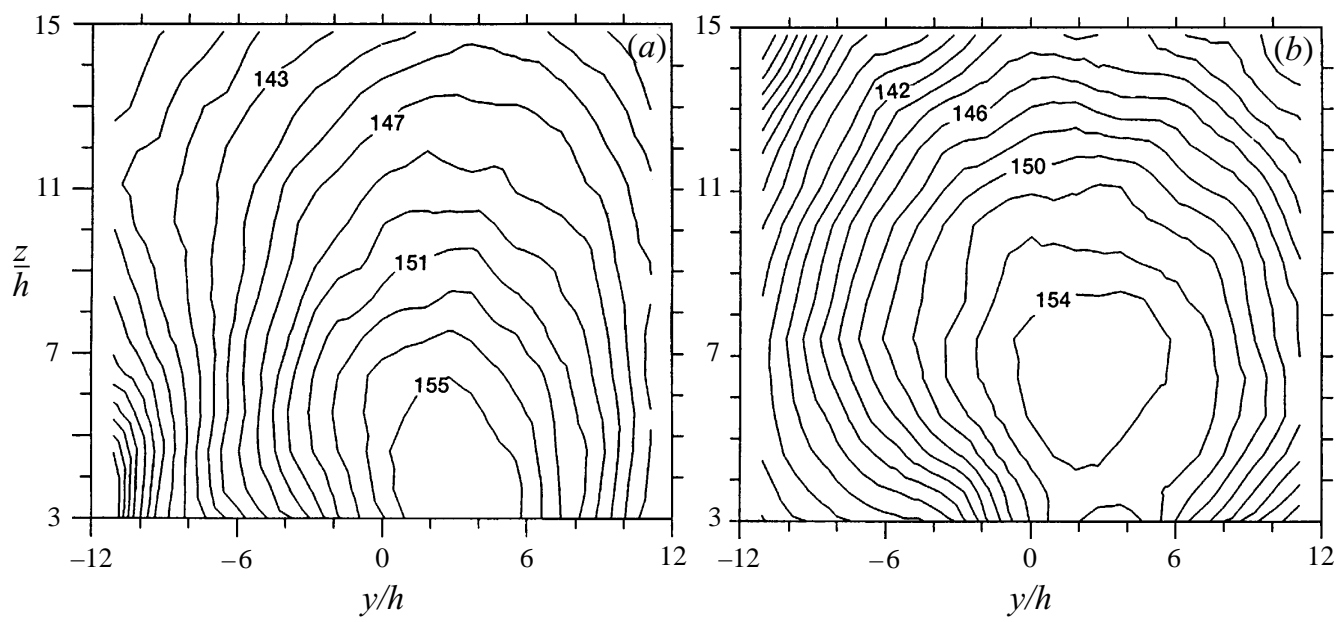

FIGURE 14. Time-averaged near acoustic field in the $(Y, Z)$-plane for single jets. $(a) M_{j}=1.48$, (b) $M_{j}=1.55$.

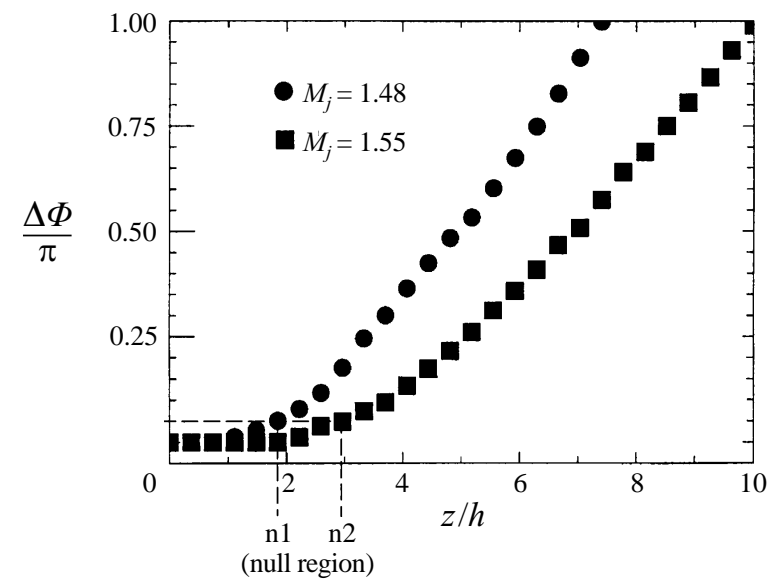

FIGURE 15. Relative phase variation in the near-nozzle region of single jets. $x / h=0, y / h=2.75$.

This null region must be accounted for in determining the coupling modes of closely spaced jets.

Tabulated experimental results for the shock spacings, acoustic wavelengths, screech source locations, and the growth of the null region of a single jet with $M_{j}$ are given in tables 1 and 2. The shock spacings (average of first five shock cells) given in table 1 agree with the predictions of Tam (1988) and Morris, Bhat \& Chen (1989). An explicit comparison is not shown since the objective here was not to check shock spacing theories but to explain the mechanism for twin-jet coupling. In table 1 the equivalent screech source was at the end of the third shock cell for $M_{j}<1.5$ and at the end of the fourth shock-cell for $M_{j}>1.5$. The source shift was derived based on the relative phase measured along a single line in the transverse direction $(z)$ at the nozzle exit plane (see Raman 1997b). Schlieren flow visualization also substantiated this finding (see Raman \& Taghavi 1996). Table 2 shows that the null region $n / h$ for single jets can grow from 1.3 at $M_{j}=1.35$ to 4.81 at $M_{j}=1.75$. Based on the null region a simple explanation can now be provided for the two modes of coupling. 


\begin{tabular}{cccccc}
\hline $\begin{array}{c}\text { Mach } \\
\text { number, } \\
M_{j}\end{array}$ & $\begin{array}{c}\text { Frequency, } \\
f(\mathrm{~Hz})\end{array}$ & $\begin{array}{c}\text { Acoustic } \\
\text { wavelength } \\
\lambda / h\end{array}$ & $\begin{array}{c}\text { Shock spacing, } \\
L_{s} / h \\
\text { (average) }\end{array}$ & $\begin{array}{c}\text { Axial distance of } \\
\text { screech source } \\
\text { from nozzle exit, } \\
q_{s} / h\end{array}$ & $\begin{array}{c}\text { Twin-jet coupling } \\
\text { mode }\end{array}$ \\
1.35 & 10200 & 4.88 & 1.849 & 5.547 & $\begin{array}{c}\text { I } \\
1.45\end{array}$ \\
1.55 & 8512 & 5.84 & 2.270 & & (antisymmetric) \\
I
\end{tabular}

From Table 2 it can be seen (if one were to imagine two jets placed side by side) that when the null regions of both jets did not overlap, mode I coupling occurred, and when they did overlap the jets coupled in mode II. Further, table 2 also shows that the sign of $\alpha((s-2 n) / h)$ determined the mode of coupling. In other words, if the nozzle spacing $(s / h$, where $h$ was the small dimension of the nozzle) was larger than twice the "null" region, we saw mode I coupling. If the inequality is reversed $(2 n>s)$, then mode II coupling was observed. Thus, although both modes of coupling are kinematically permissible, the choice of coupling mode depends on the size of the 'null' region relative to the nozzle spacing (see sketch in table 2).

\subsection{Mode switch mechanism and transition}

In $\S 3.4$ we documented the abrupt switch from mode I to II. A question that naturally arises is what causes the abrupt mode switch? Our explanation is that at $M_{j}=1.5$ there was an abrupt shift in the equivalent source of screech from the third to the fourth shock cell that caused an abrupt growth of the null region. Proof of the source shift and a model for the growth of the null region for single jets was provided by Raman \& Taghavi (1996) and Raman (1997b) and will not be reiterated here. However, we wish to point out that although there were numerous sources, the effective source centre (for rectangular jets) depended on only one or two shock cells (third or fourth) depending on the jet Mach number (Raman 1997b). The abrupt growth of the null region at $M_{j}=1.5$ in turn caused the null regions of both jets to overlap (negative $\alpha$ ) and produced an abrupt switch from mode I to II. 

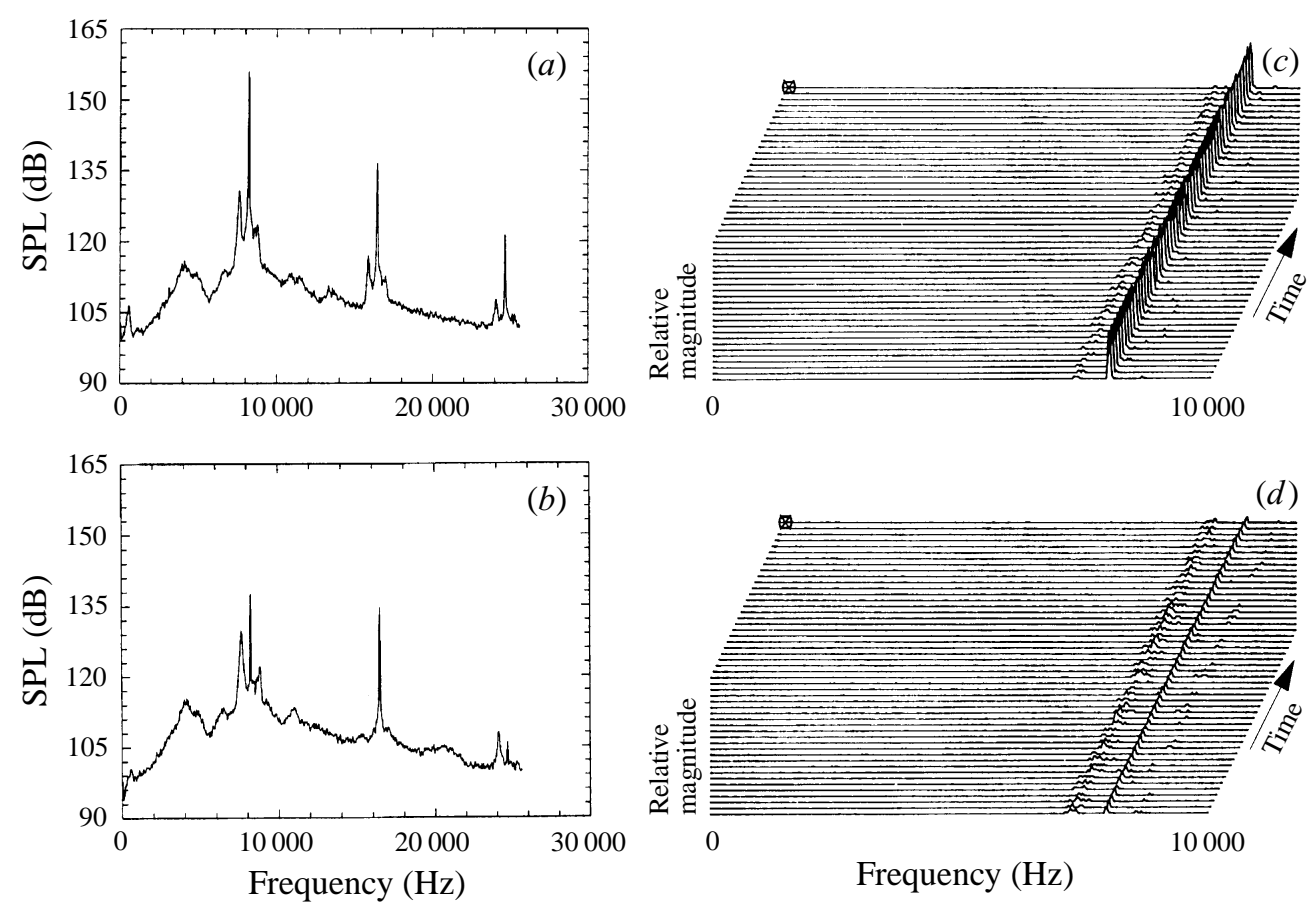

FiguRE 16. Steadiness of twin jets coupled in mode I. $s / h=5.5, M_{j}=1.48$. (a) Time-averaged spectrum from microphone mounted on nozzle $1(x / h=0, y / h=-2.75, z / h=1),(b)$ time-averaged spectrum from microphone mounted in between nozzles 1 and $2(x / h=0, y / h=0, z / h=1),(c, d)$ instantaneous spectra for the frequency range from $0-10000 \mathrm{~Hz}$, microphone locations the same as that for cases $(a, b)$, respectively.

For jets that were coupled it was also important to address the issue of steadiness and transition from one mode to another. Figure 16(a,b) represents time-averaged spectra for mode I coupling from a microphone located on nozzle $1(x / h=0$, $y / h=-2.75, z / h=1)$ and another located mid-way between the two nozzles $(x / h=0, y / h=0, z / h=1)$. The amplitude of the dominant coupled mode was higher at $y / h=-2.75$ than at $y / h=0$ as shown earlier in figure $7(a)$. The 'instantaneous spectra' shown in figure $16(c, d)$ address the steadiness issue. The instantaneous spectra were obtained by taking a long time sequence and performing an FFT on smaller segments. The resulting plot shows the variation of spectra with time. One limitation of the instantaneous spectra was that 1024 samples were required for each line in the plot. The sampling of 1024 points even at $200 \mathrm{kHz}$ required $5.12 \times 10^{-3} \mathrm{~s}$; with a $40 \%$ overlap between consecutive lines in the instantaneous spectra this time reduced to $3.072 \times 10^{-3} \mathrm{~s}$. For a typical screech signal of $7000 \mathrm{~Hz}$ frequency the time scale was $1.43 \times 10^{-4}$ seconds which was an order of magnitude lower than the resolution scale of the instantaneous spectra. Thus, each line in the instantaneous spectrum included numerous screech cycles. Nevertheless this technique was still of limited use in resolving issues pertaining to steadiness. Going back to figure $16(c, d)$ which zooms in on the region from 0 to $10 \mathrm{kHz}$ one can see that the dominant coupling frequency was steady in time. In comparison, a frequency that was slightly lower than the dominant frequency was unsteady in figure $16(c, d)$. Similar observations can be made from figure $17(a-d)$ for mode II coupling. However, a difference between figures 16 and 17 is that mode II coupling (figure 17) produced levels that were 

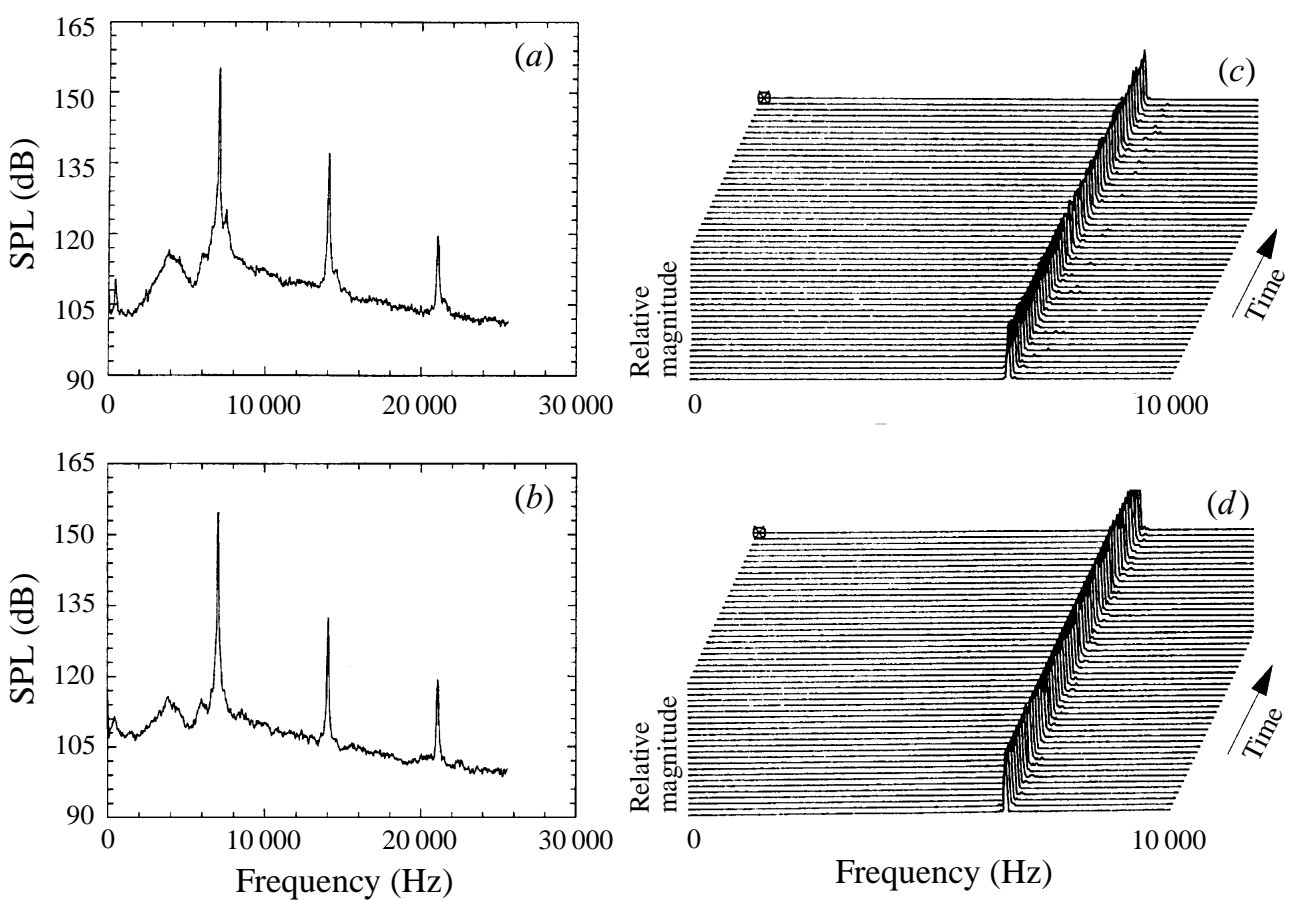

FIgURE 17. Steadiness of twin jets coupled in mode II. $s / h=5.5, M_{j}=1.55$. Parts $(a-d)$ as described in the caption for figure 16 .

higher at $y / h=0$ (figure $17 b$ ) than at $y / h=-2.75$ (figure 17a). The reverse was true for figure 16. Note that had the coupling not been steady for modes I and II (figures 16 and 17) we would not have been able to make the detailed phase-averaged measurements described in $\$ 3.3$.

Finally, we approach the issue of mode transition. It is of interest to know if modes I and II are mutually exclusive or can both coexist at the transition point. A further point of interest is whether the modes switch back and forth before the jets make a final selection. The data of figure 18 were taken while increasing the Mach number from 1.497 (Mode I) to 1.510 (Mode II). The time-averaged spectrum (figure 18a) from the microphone on nozzle 1 indicated the presence of both modes. In contrast, the instantaneous spectra indicated that a switch occurred from mode I to II, and that both modes did not coexist. (Even when the jet was operated at the mode switch point, the two coupling modes never coexisted.) This technique was used earlier (Raman 1997a) to spot mutually exclusive switching or the co-existence of two modes in jets from bevelled nozzles.

Note that the mode switch is also accompanied by a frequency jump to a lower frequency (also seen previously in figure $6 a$ ). The microphone located in between the two nozzles (figure $18 b, d$ ) showed some interesting features. The time-averaged spectrum (figure 18b) showed a high-amplitude mode II and a low-amplitude mode I, agreeing with the data of figure 7 (i.e. mode I always exhibited a minimum in the inter-nozzle region). The instantaneous spectrum of figure $18(d)$ indicated the transition from low pressure levels (mode I) to higher levels (mode II). It is of interest to see if the reverse happened when the Mach number was reduced from 1.510 (mode II) to 1.497 (mode I). Figure 19( $a-d)$ shows that this was indeed the case. Hysteresis, if any, was not pronounced. 

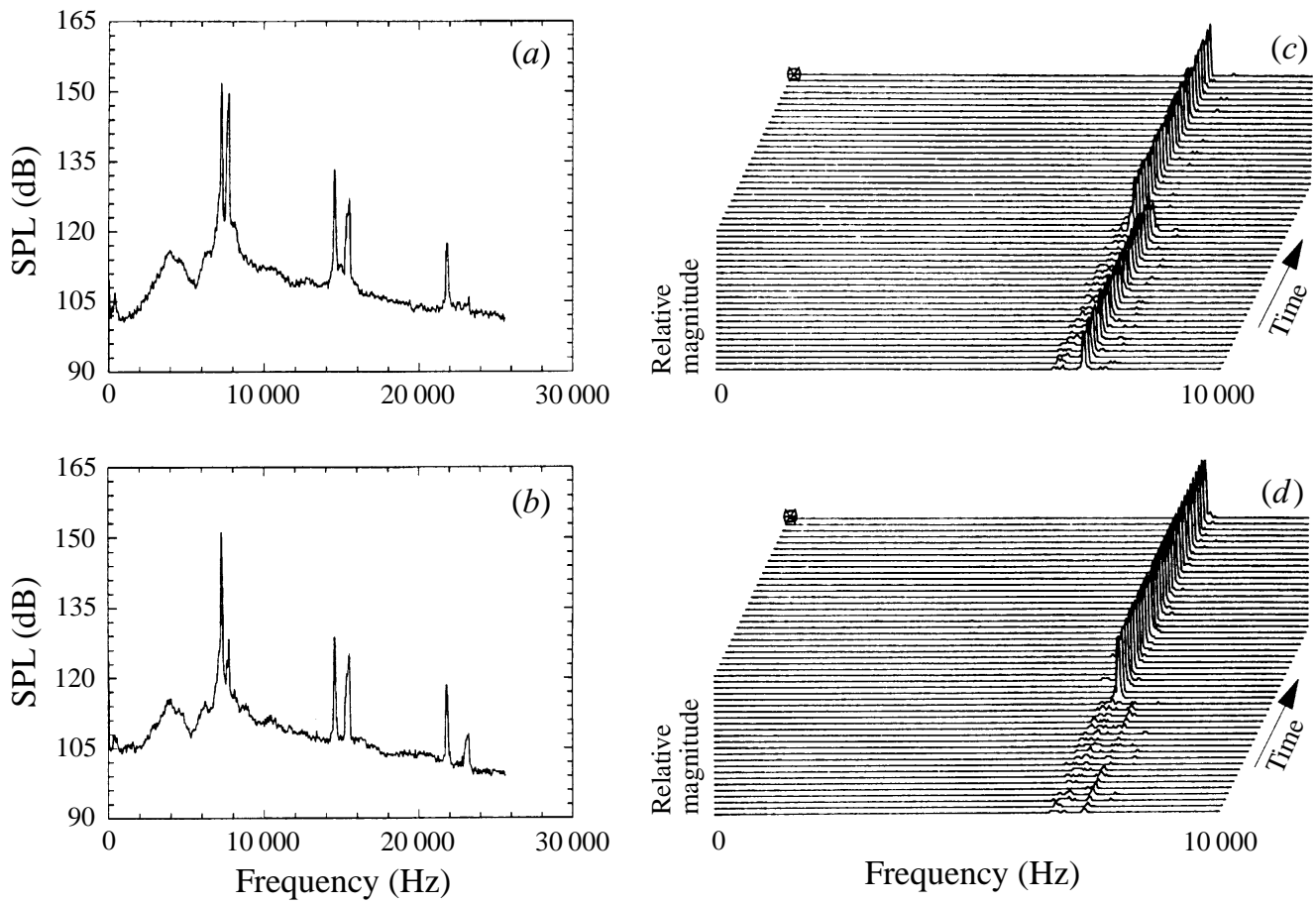

FIGURE 18. Transition from mode I to II, $s / h=5.5, M_{j}$ increased from 1.48 to 1.55 . Parts $(a-d)$ as described in the caption for figure 16.
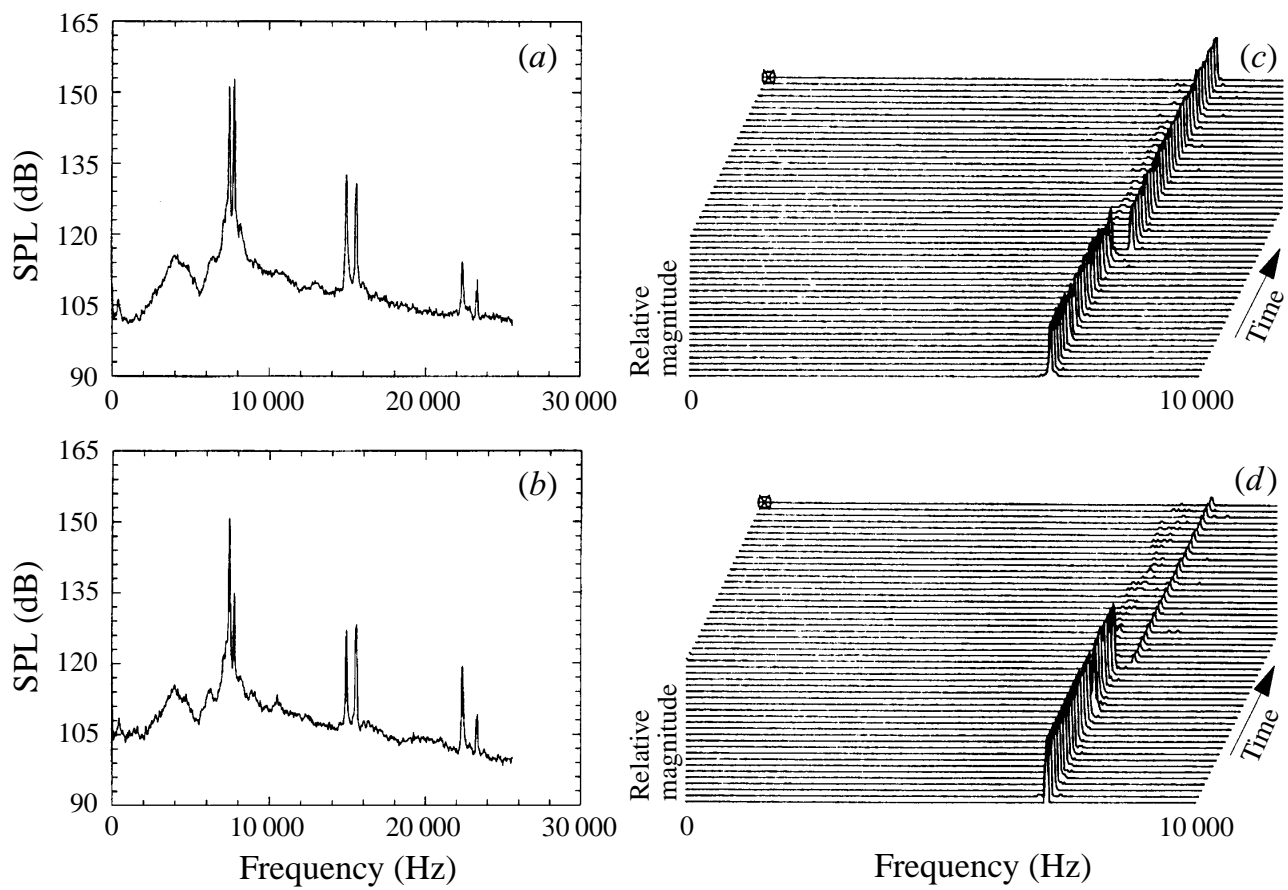

FIGURE 19. Transition from mode II to I, $s / h=5.5, M_{j}$ decreased from 1.55 to 1.48 . Parts $(a-d)$ as described in the caption for figure 16. 


\section{Summary and conclusions}

The twin-rectangular-jet problem is important in closely spaced twin-engine aircraft. We explored details of the near acoustic field and coupling mechanisms. It was found that if the jets had slightly different frequencies, the one with the higher screech amplitude dominated and influenced the frequency of the coupled jets. Antisymmetric coupling produced minimum pressure in the inter-nozzle region, whereas symmetric coupling produced maximum pressure in the same region. Phase-locked motions were observed and were found to persist over the entire screech cycle. We determined the ensemble-averaged periodic pressure loadings and finally documented the transition from the antisymmetric to the symmetric mode.

We focused on answering the three questions raised in \$1.2. Our results indicate the following. (a) For very closely spaced twin jets in the side-by-side configuration phased feedback based on source to nozzle exit distance of adjacent jets does not fully explain the coupling modes. The null region surrounding the jets where the acoustic wavefronts arriving from downstream are flat over a small radial distance appears to influence the mode of coupling. We provide a parameter $(\alpha)$ that can be used as a simple test to determine the mode of coupling. (b) The switch from the antisymmetric to the symmetric mode of coupling appears to occur because of an abrupt shift in the effective screech source from the third to the fourth shock, which in turn causes the null phase region surrounding the jets to grow abruptly and overlap. (c) The two observed modes were mutually exclusive. The data presented provide considerable insight into the twin jet coupling problem even though we have not been able to answer all questions regarding this complicated problem.

A version of this paper was presented at the 3rd AIAA/CEAS Aeroacoustics conference. The authors are grateful to Professor C. K. W. Tam of Florida State University for providing insightful comments. Dr Steven Walker of the US Air Force (Wright Laboratory) and Dr Alan Cain of McDonnell Douglas Aerospace encouraged and motivated this work. The authors thank Dr J. Panda for providing a computer routine for phase-averaged data acquisition and Mr Ken Weiland and Dr Carolyn Mercer for their schlieren expertise.

\section{REFERENCES}

BERndT, D. E. 1984 Dynamic pressure fluctuations in the internozzle region of a twin-jet nacelle. SAE Paper 84-1540. SAE Aerospace Cong. \& Expo., Long Beach, CA.

CAIn, A. B. \& Bower, W. W. 1996 Modeling supersonic jet screech: differential entrainment and amplitude effects. AIAA Paper 96-0916.

Cain, A. B., Bower, W. W., Walker, S. H. \& Lockwood, M. K. 1995 Modeling supersonic jet screech. Part 1: vortical instability wave modeling. AIAA Paper 95-0506.

HARPER-Bourne, M. \& Fisher, M. J. 1973 The noise from shock waves in supersonic jets. Noise mechanisms. AGARD CP 131, pp. 11-1-11-13.

Hay, J. A. \& Rose, E. G. 1970 In-flight shock cell noise. J. Sound Vib. 11, 411-420.

Morris, P. J. 1990 Instability waves in twin supersonic jets. J. Fluid Mech. 220, 293-307.

Morris, P. J., Bhat, T. R. S. \& Chen, G. 1989 A linear shock cell model for jets of arbitrary exit geometry. J. Sound Vib. 132, 199-211.

Norum, T. D. \& Shearin, J. G. 1986 Dynamic loads on twin jet exhaust nozzles due to shock noise. J. Aircraft 23, 728-729.

PANDA, J. 1996 An experimental investigation of screech noise generation. AIAA Paper 96-1718.

Powell, A. 1953 On the mechanism of choked jet noise. Proc. Phys. Soc. Lond. 66, 1039-1056.

RAMAN, G. $1997 a$ Screech tones from rectangular jets with spanwise oblique shock cell structures. J. Fluid Mech. 330, 141-168. 
Raman, G. $1997 b$ Cessation of screech in underexpanded jets. J. Fluid Mech. 336, 69-90.

Raman, G. \& TaghaVi, R. 1996 Resonant interaction of a linear array of supersonic rectangular jets: an experimental study. J. Fluid Mech. 309, 93-111 (and Corrigendum 326, 1996, 437).

RicE, E. J. 1995 Jet mixer noise suppressor using acoustic feedback. US Patents 5,325,661, and $5,392,597$.

Seiner, J. M., Manning, J. C., Capone, F. J. \& Pendergraft, JR., O. C. 1992 Study of external dynamic flap loads on a 6 percent B-1B model. J. Eng. Gas Turbines and Power, 114, 817-828.

Seiner, J. M., Manning, J. C. \& Ponton, M. K. 1988 Dynamic pressure loads associated with twin supersonic plume resonance. AIAA J. 26, 954-960.

SHaw, L. 1990 Twin-jet screech suppression. J. Aircraft, 27, 708-715.

TAM, C. K. W. 1988 The shock-cell structures and screech tone frequencies of rectangular and non-axisymmetric supersonic jets. J. Sound Vib. 121, 135-147.

TAM, C. K. W. 1991 Jet noise generated by large-scale coherent motion. In Aeroacoustics of Flight Vehicles: Theory and Practice, Vo1. 1: Noise sources (ed. H. H. Hubbard). NASA RP 1258, WRDC TR 90-3052, pp. 311-390.

TAM, C. K. W. \& Seiner, J. M. 1987 Analysis of twin supersonic plume resonance. AIAA Paper 87-2695.

TAM, C. K. W. \& Shen, H. 1998 Numerical simulation of the generation of axisymmetric mode jet screech tones. AIAA Paper 98-0283.

TAM, C. K. W. \& Thies, A. T. 1993 Instability of rectangular jets. J. Fluid Mech. 248, 425-448.

WALKER, S. H. 1990 Twin jet screech suppression concepts tested for $4.7 \%$ axisymmetric and two-dimensional nozzle configurations. Rep. WRDC-TR-90-3063. Wright-Patterson Air Force Base, USA.

Walker, S. H., Gordeyev, S. V. \& Thomas, F. O. 1995 A wavelet transform analysis applied to unsteady jet screech resonance. In High Speed Jet Flows (ed. G. Raman, S. Kaji \& C. Freitas), ASME FED Vol. 214, 103-108.

Westley, R. \& Woolley, J. H. 1969 The near field sound pressures of a choked jet during a screech cycle. Aircraft engine noise and sonic boom. AGARD CP 42, 23-1-23-13.

Westley, R. \& Woolley, J. H. 1970 The near field sound pressures associated with a spinning screech mode. Conference on Current Developments in Sonic Fatigue. Institute of Sound and Vibration, University of Southampton, England.

Wlezien, R. W. 1987 Nozzle geometry effects on supersonic jet interaction. AIAA Paper 87-2694.

Zilz, D. E. \& Wlezien, R. W. 1990 The sensitivity of near-field acoustics to the orientation of twin two-dimensional supersonic nozzles. AIAA Paper 90-2149. 